\title{
Synthesis of new aryl substituted 5-alkylidenefuran-2(5H)-ones
}

\author{
Ruonan Zhang, Daniel Chan, Shari Jessica, George Iskander, David StC Black, and \\ Naresh Kumar* \\ School of Chemistry, University of New South Wales, UNSW, Sydney, NSW 2052, Australia \\ E-mail:n.kumar@unsw.edu.au
}

\section{Dedicated to Professor Ted Sorenson}

\begin{abstract}
The rapid emergence of bacterial resistance in recent years has demanded the development of new drugs and therapies with novel modes of action to supplement existing antibiotics. Brominated furanones or fimbrolides are a promising class of quorum sensing inhibitors that inhibit biofilm formation and the expression of virulence in in vitro and in vivo models. We report here an efficient synthesis of novel brominated 5-alkylidenefuran-2(5H)-ones, making use of $\gamma$-oxo- $\alpha, \beta$-dibromoalkanoic acid 7 as the key intermediate. Brominated furanones were subjected to the Suzuki-Miyaura cross-coupling reaction to generate a library of novel aryl furanones. This methodology may be readily adapted to the synthesis of a wide variety of substituted furanones.
\end{abstract}

Keywords: Furanones, 5-alkylidenefuranones, anti-bacterial, Suzuki-Miyaura reaction, dibromoalkanoic acids, alkenoic acids

\section{Introduction}

The emergence of multi-resistant strains of bacteria in recent years has driven the search for new therapeutic compounds with novel modes of action to supplement existing anti-microbials. ${ }^{1}$ Traditional antibiotics impose selective pressure on bacteria, leading to drug resistance. ${ }^{2} \mathrm{~A}$ new key strategy is to target the various regulatory systems in bacteria that control the expression of virulence.

Bacteria communicate with each other via small intercellular signal compounds and this regulatory system is known as quorum sensing (QS). ${ }^{3,4}$ Quorum sensing is critical for bacterial biofilm formation and the expression of virulent phenotypes. $N$-Acyl-L-homoserine lactones (AHL) are the most widely studied class of QS mediators and they are used by many pathogenic Gram-negative bacterial species. ${ }^{5}$ 
Natural furanones $\mathbf{1}$ isolated from the red alga Delisea pulchra were found to possess significant QS inhibitor activity (Figure 1). ${ }^{6}$ They share a common 4-halo-3-butyl-5halomethylenefuran-2(5H)-one skeleton but vary in the number and nature of the halogen substituents and the presence or absence of oxygen functionality in the butyl side-chain. These halogenated furanones or "fimbrolides" inhibit biofilm formation by specifically interfering with AHL-mediated quorum sensing. Importantly, the expression of the virulent phenotypes was inhibited at non-bactericidal concentrations. ${ }^{7-10}$ Therefore, these furanones should not impose the same selective pressure as conventional antibiotics on bacterial strains to develop resistance. To date over 200 analogues of furanones with different bromination patterns and alkyl chain lengths have been generated and evaluated for QS inhibitor activities. ${ }^{11}$

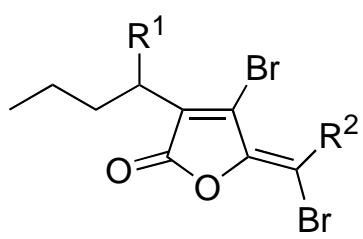

1

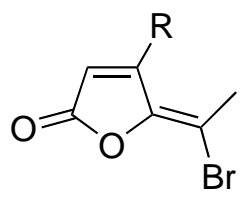

2

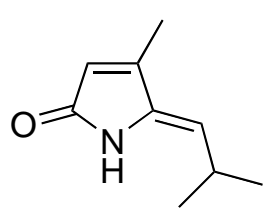

3

$$
\begin{aligned}
& \mathrm{R}^{1}=\mathrm{H}, \mathrm{OH}, \mathrm{OAC} \quad \mathrm{R}=\mathrm{H}, \mathrm{Br} \\
& \mathrm{R}^{2}=\mathrm{H}, \mathrm{Br}
\end{aligned}
$$

Figure 1. Natural fimbrolides 1 from D. pulchra, synthetic fimbrolides 2 and pulchellalactam 3.

Studies have shown that simple fimbrolides 2 lacking the C3-butyl still show significant QS inhibitor activity. ${ }^{8,10}$ Synthetic fimbrolide $2(\mathrm{R}=\mathrm{Br})$ was found to reduce $P$. aeruginosa levels in the lungs by three orders of magnitude in a pulmonary mouse model. ${ }^{9}$

Intrigued by the exocyclic iso-propyl moiety in the structurally related natural product pulchellalactam ${ }^{12} 3$ as well as the presence of the similar tert-butyl group in various antibacterial compounds, ${ }^{13,14}$ we set out to synthesize brominated furanones $4\left(\mathrm{R}^{1}\right.$ or $\left.\mathrm{R}^{2}=\mathrm{Br}\right)$ carrying a vinylic iso-propyl or tert-butyl substituent at the $\mathrm{C} 5$ position (Figure 2). Compounds where the C4-bromine was switched to the $\mathrm{C} 3$ position were also targeted to afford a more comprehensive library of furanones for structure-function investigations. 


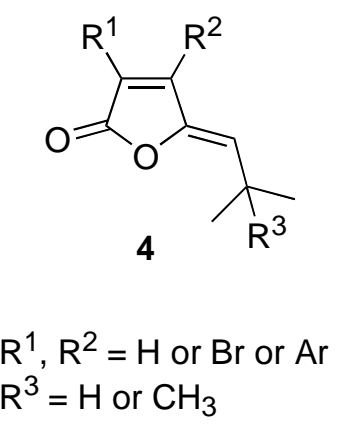

Figure 2. Targeted furanones 4.

We also envisaged that these brominated furanones could be utilized as synthetic intermediates in the Suzuki-Miyaura cross-coupling reaction. ${ }^{15}$ 3-Aryl- and 4-arylfuran-2(5H)one cores are widely distributed in natural products and biologically active compounds. ${ }^{16,17}$ Consequently, the synthesis of novel 3- and 4-aryl-5-alkylidenefuranones $4\left(\mathrm{R}^{1}\right.$ or $\left.\mathrm{R}^{2}=\mathrm{Ar}\right)$ was of interest to us.

\section{Results and Discussion}

A variety of methods have been reported in the literature for the synthesis of fimbrolides and their analogues. ${ }^{11}$ However, most approaches tended to be unwieldy and required many steps in the synthetic sequence. The most facile synthesis of our targeted fimbrolides used a methodology developed by our group involving the key intermediate $\gamma$-oxo- $\alpha, \beta$-dibromoalkanoic acid 7 (Scheme 1). ${ }^{18}$ Unbranched alkanones related to compounds 5 undergo reaction with glyoxylic acid at the methylene rather than the methyl group adjacent to the carbonyl: however, alkanones 5 react regioselectively at the methyl group. 


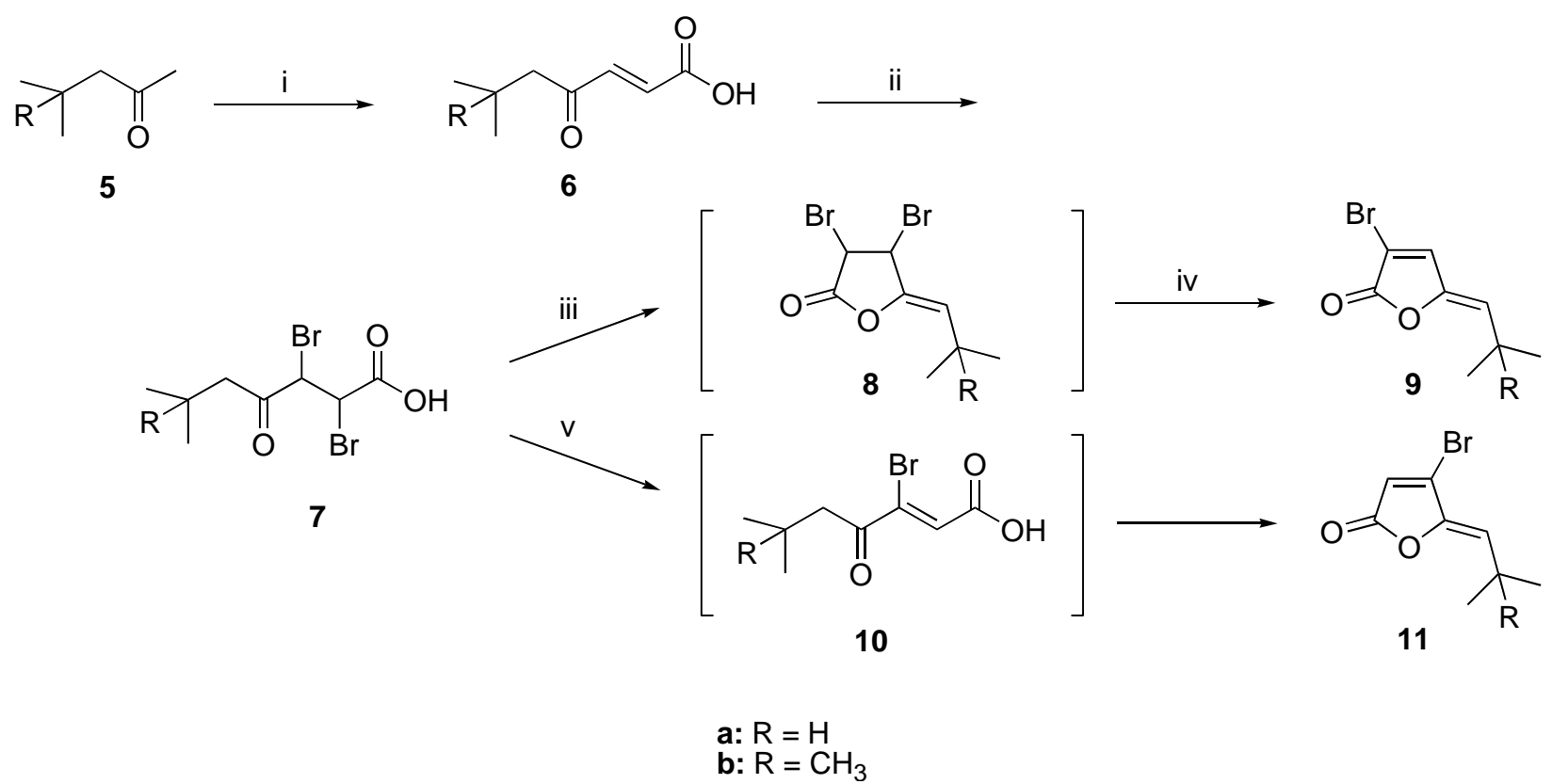

Scheme 1. Reagent and conditions: (i) $\mathrm{HCOCOOH} \mathrm{(1.6} \mathrm{equiv),} \mathrm{H}_{3} \mathrm{PO}_{4}, 75{ }^{\circ} \mathrm{C}, 5 \mathrm{~h}(\mathbf{6 a}: 60 \%$, $6 \mathbf{b}$ : 40\%); (ii) $\mathrm{Br}_{2}$ (0.9 equiv), $\mathrm{CH}_{2} \mathrm{Cl}_{2},-78{ }^{\circ} \mathrm{C}, 3 \mathrm{~h}$ (7a: 78\%, 7b: 89\%); iii) $\mathrm{P}_{2} \mathrm{O}_{5}, \mathrm{CH}_{2} \mathrm{Cl}_{2}$, reflux, 1 h; (iv) DBU (1.1 equiv), $\mathrm{CH}_{2} \mathrm{Cl}_{2},-78{ }^{\circ} \mathrm{C}, 1 \mathrm{~h}$ (9a: 63\%, 9b: 75\%); (v) $\mathrm{H}_{2} \mathrm{SO}_{4}, \mathrm{CH}_{2} \mathrm{Cl}_{2}, 100{ }^{\circ} \mathrm{C}$, reflux (11a: 54\%, 11b: 25\%).

The first step involved the regioselective condensation of alkanones 5 with glyoxylic acid. The ${ }^{1} \mathrm{H}$ NMR spectrum of the alkenoic acid 6a revealed two doublets at $\delta 5.59$ and $7.09 \mathrm{ppm}$ with coupling constant $15.8 \mathrm{~Hz}$, confirming the regioselective condensation at the $\mathrm{C} 1$ position with $(E)$-stereochemistry at the double bond. The minor C3 regioisomer was also detected in small yield, but could be removed by recrystallization from light petroleum. In the case of alkenoic acid $\mathbf{6 b}$, only the $\mathrm{C} 1$ regioisomer was formed, presumably due to the greater steric influence of the bulkier tert-butyl group. Again, the doublets at $\delta 6.58$ and $7.08 \mathrm{ppm}(\mathrm{J}=16.0 \mathrm{~Hz})$ indicated the formation of the desired $E$-isomer.

Bromination of the alkenoic acids 6 provided the key dibromo acid intermediates 7 in 78 $89 \%$ yield. This was evidenced by the disappearance of the olefinic doublets and the rise of a new pair of doublets at $\delta 4.60-4.64$ and 4.69-4.72 ppm with a much smaller coupling constant of $J$ 1.1-2.6 Hz. The expected anti-conformation of acid 7a was confirmed by spectroscopic data.

Dehydration of the dibromo intermediates 7 with phosphorus pentoxide gave the 3,4dibromo-dihydrofuran-2-ones 8 as yellow oils. These compounds proved to be difficult to isolate and purify, therefore the crude material was treated directly with 1,8-diazabicyclo[5.4.0] undec-7ene (DBU) to afford 3-bromofuranones 9 in 63-75\% yield. The dehydrobromination reactions proceeded with high regioselectivity and the minor 4-bromofuranone products were not observed. In the proton spectrum of furanone 9a, the olefinic proton of the methylpropylidene group resonated as a doublet at $\delta 5.19 \mathrm{ppm}$ while the $\mathrm{H} 4$ proton appeared as a singlet at $\delta 7.39 \mathrm{ppm}$. 
These two protons coupled to each other in the 2D NOESY NMR spectrum confirming the Zstereochemistry of the double bond (Figure 3). For 3-bromofuranone $\mathbf{9 b}$, the two protons appeared as singlets at $\delta 5.26$ and 7.35 ppm, respectively.

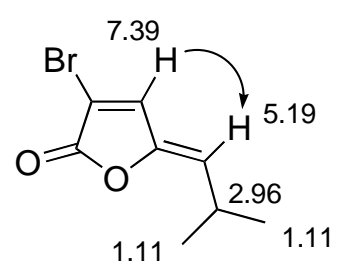

$9 \mathbf{a}$

Figure 3. NOESY coupling in fimbrolide 9a.

In contrast, the acid-promoted cyclization of the dibromo intermediates 7 gave the 4bromofuranone 11 in $25-54 \%$ yield in a single reaction. This reaction is postulated to proceed via the dehydrobromination intermediate 10, which then undergoes cyclization and dehydration to yield 4-bromofuranone 11. This is consistent with observations in related examples. ${ }^{18}$ In the ${ }^{1} \mathrm{H}$ NMR of furanone 11a, the olefinic proton of the methylpropylidene group resonated as a doublet at $\delta 5.44 \mathrm{ppm}$, while $\mathrm{H} 3$ appeared as a singlet $\delta 6.32 \mathrm{ppm}$. For furanone 11b, these protons appeared as singlets at $\delta 5.54$ and 6.28 ppm respectively. Although 2D NMR could not establish the stereochemistry of the double bond in 4-bromofuranones, the chemical shift values of these protons were similar to those in the naturally occurring ( $Z$ )-fimbrolides. Furthermore, the bulky iso-propyl and tert-butyl groups would be expected to strongly discourage the formation of the $E$-isomer due to steric clashes with the $\mathrm{C} 4$ bromine.

This optimized sequence required only one chromatographic purification to furnish the desired 3-bromo or 4-bromofuranones in three to four steps from inexpensive starting materials. This methodology may be readily adapted to the synthesis of a wide range of substituted furanone analogues. Preliminary screening results have demonstrated that some of these synthetic furanones possess AHL-inhibitory activity.

The Suzuki-Miyaura reaction has previously been reported for the synthesis of 3 -aryl ${ }^{16}$ and 4-arylfuran-2 $(5 \mathrm{H})$-ones ${ }^{17}$ lacking the $\mathrm{C} 5$ exocyclic double bond, while for fimbrolide analogues the Stille coupling has been tried. ${ }^{19}$ Iodinated fimbrolide-like structures have also been subjected to the Suzuki reaction for the synthesis of complex natural products. ${ }^{20}$ However, this appears to be the first time that the Suzuki reaction has been applied to close analogues of the natural fimbrolides.

The Suzuki-Miyaura coupling reactions of the 3-bromofuranone $\mathbf{9 b}$ were realized in a biphasic toluene-water (1:1) solvent system with $\mathrm{Pd}\left(\mathrm{PPh}_{3}\right) \mathrm{Cl}_{2}$ as catalyst, $\mathrm{CsF}$ as base and $\mathrm{Bu}_{4} \mathrm{NI}$ as a phase transfer agent. A variety of boronic acids 12a-i were investigated to give a library of new 3-aryl-5-alkylidenefuran-2(5H)-ones 13a-i (Table 1).

The identity of the coupling products was confirmed by ${ }^{1} \mathrm{H}$ and ${ }^{13} \mathrm{C} \mathrm{NMR}$, HRMS, and elemental analyses. The Z-stereochemistry of the exocyclic double bond can be clearly seen from 
the crystal structure of 3-(3-fluorophenyl)-5-(2,2-dimethylproprylidene)furan-2(5H)-one 13c (Figure 4).

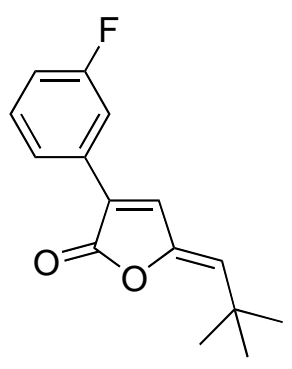

13c

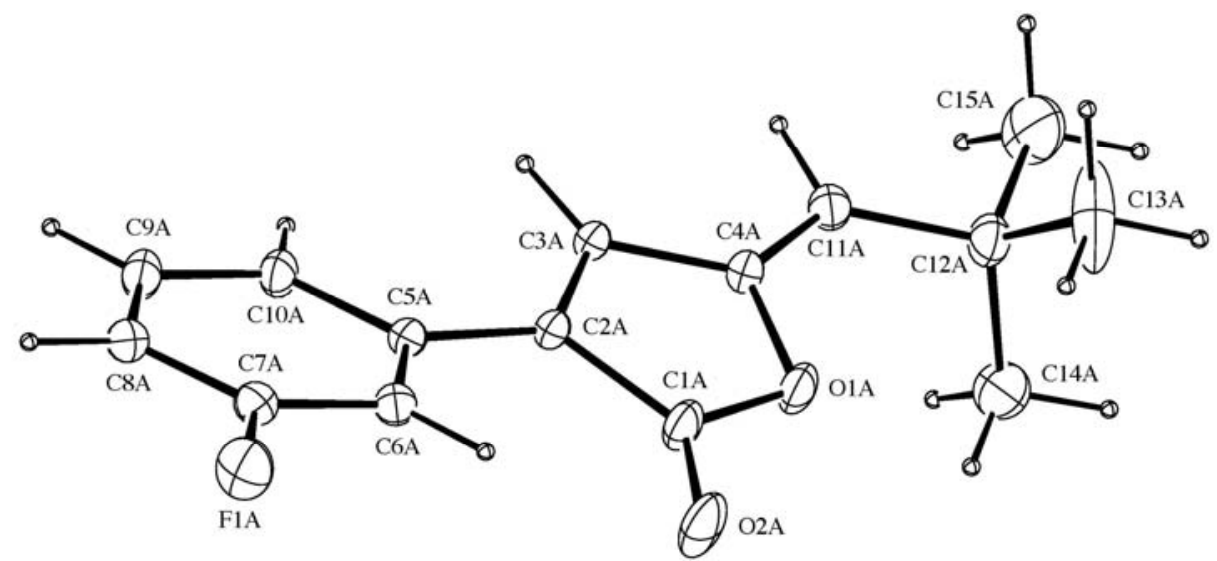

Figure 4. X-ray crystal structure of furanone 13c.

In the ${ }^{1} \mathrm{H}$ NMR of the 3-arylfuranones, the exocyclic vinylic proton appeared in the range $\delta$ 5.20-5.40 ppm and $\mathrm{H} 4$ at $\delta$ 7.34-7.65 ppm, while in the starting material 3-bromofuranone 9b these protons resonated $\delta 5.26$ and 7.35 ppm, respectively. Generally, the substitution of an aryl group for the bromine atom results in a downfield shift for these protons. 
Table 1. Suzuki-Miyaura coupling of 3-bromofuranone $9 \mathbf{b}$ with boronic acids $12 a-\mathbf{i}^{\mathrm{a}}$<smiles>CC(C)(C)/C=C1/C=C(Br)C(=O)O1</smiles>

$9 b$

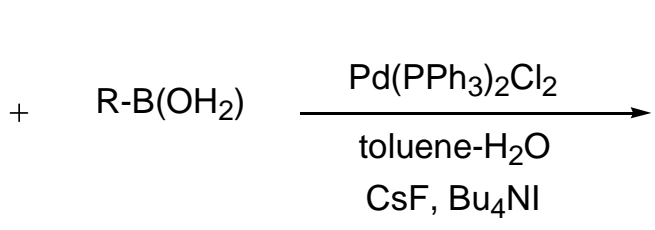

12

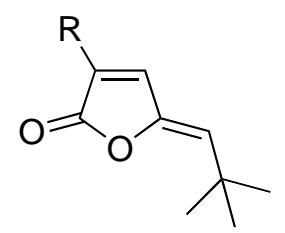

13

\begin{tabular}{|c|c|c|c|c|}
\hline Entry & 12 & $\mathrm{R}$ & Product 13 & Yield $^{\mathrm{b}}(\%)$ \\
\hline 1 & $12 a$ & & $13 a$ & 89 \\
\hline 2 & $12 b$ & & $13 b$ & 39 \\
\hline 3 & $12 c$ & & $13 c$ & 66 \\
\hline 4 & 12d & & 13d & 74 \\
\hline 5 & $12 e$ & & 13e & 53 \\
\hline 6 & $12 f$ & & $13 f$ & 61 \\
\hline 7 & $12 \mathrm{~g}$ & & $13 g$ & 70 \\
\hline 8 & $12 \mathrm{~h}$ & & $13 h$ & 45 \\
\hline 9 & $12 \mathbf{i}$ & & $13 \mathbf{i}$ & 21 \\
\hline
\end{tabular}

${ }^{a}$ Reagents and conditions: $\mathrm{R}-\mathrm{B}(\mathrm{OH})_{2}$ (1.2 equiv), $\mathrm{CsF}$ ( 3 equiv), $\mathrm{Pd}\left(\mathrm{Ph}_{3}\right) \mathrm{Cl}_{2}\left(0.05\right.$ equiv), $\mathrm{Bu}_{4} \mathrm{NI}$ ( 0.05 equiv), reflux, $36 \mathrm{~h}$.

${ }^{\mathrm{b}}$ Isolated yields. 
Similarly, reaction of 4-bromofuranone $\mathbf{1 1 b}$ with boronic acids 12b-d gave 4-aryl-5alkylidenefuran-2(5H)-ones 14a-c (Scheme 2).

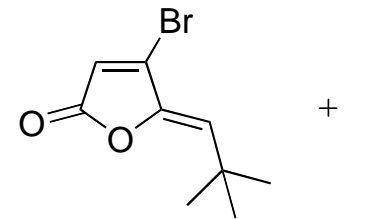

11b

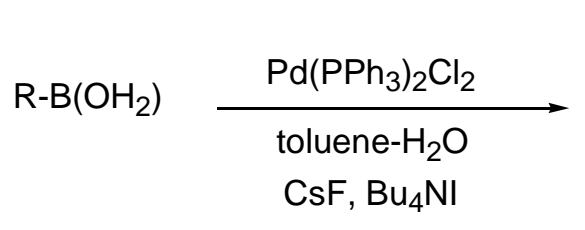

12

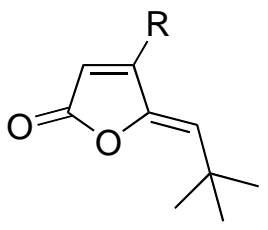

14

a: $\mathrm{R}=4$-fluorophenyl (70\%)

b: $\mathrm{R}=3$-fluorophenyl (25\%)

c: $R=2$-fluorophenyl $(25 \%)$

Scheme 2. Reagents and conditions: $\mathrm{R}-\mathrm{B}(\mathrm{OH})_{2}$ (1.2 equiv), $\mathrm{CsF}$ (3 equiv), $\mathrm{Pd}\left(\mathrm{Ph}_{3}\right) \mathrm{Cl}_{2}(0.05$ equiv), $\mathrm{Bu}_{4} \mathrm{NI}(0.05$ equiv), reflux, $36 \mathrm{~h}$.

\section{Conclusions}

In summary, we have successfully prepared fimbrolide analogues that have an iso-propyl or tertbutyl substituent instead of a bromine atom at the exocyclic vinylic position. By employing different cyclization conditions for the key dibromo acid intermediate, both 3- and 4bromofuranones could be formed. The syntheses require only a few simple steps from simple starting materials and the products are obtained with high stereo- and regioselectivies. The Suzuki-Miyaura reaction was employed to generate 3- and 4-aryl analogues. Overall, the methodologies described represent an efficient access to a range of natural products and their analogues with potentially interesting biological activities.

\section{Experimental Section}

General Procedures. Melting points were determined on a Köfler hot stage apparatus and are uncorrected. ${ }^{1} \mathrm{H}$ NMR spectra were recorded with a Bruker DPX300 (300 MHz) spectrometer and NMR spectral data are reported as follows: chemical shift measured in parts per million $(\mathrm{ppm})$ internally referenced to solvent nuclei $(\delta)$; multiplicity; observed coupling constant $(J)$ in Hertz (Hz); proton count; assignment. Multiplicities are recorded as singlet (s), doublet (d), triplet $(\mathrm{t})$, quartet $(\mathrm{q})$, multiplet $(\mathrm{m})$ and combinations of these. ${ }^{13} \mathrm{C}$ NMR spectra were recorded with a Bruker DPX300 spectrometer $(75 \mathrm{MHz})$ and chemical shifts are reported in ppm internally referenced to solvent nuclei $(\delta)$, and identifiable carbons are given. Ultraviolet spectra were measured on a Cary 100 spectrophotometer and data are reported as wavelength $(\lambda)$ in $\mathrm{nm}$ and absorption coefficient $(\varepsilon)$ in $\mathrm{M}^{-1} \mathrm{~cm}^{-1}$ in the specified solvent. Infrared spectra were obtained 
with a Nicolet Avatar 320 FT-IR spectrometer on KBr discs. Elemental analyses and high resolution mass spectra were performed by the Campbell Microanalytical Laboratory, University of Otago, New Zealand. Flash column chromatography was performed using Merck 60 Silica Gel. X-ray crystal structures were obtained by Don Craig at The University of New South Wales, Sydney, Australia. Crystallography data can be obtained free of charge from The Cambridge Crystallographic Data Centre as number CCDC 692618 (13c) via www.ccdc.cam.ac.uk/data_request/cif. Reagents and solvents were purchased from standard suppliers and were used without further purification.

(E)-6-Methyl-4-oxohept-2-enoic acid (6a). To a solution of glyoxylic acid (27.61 g, $0.30 \mathrm{~mol}$ ) in ortho-phosphoric acid $(20 \mathrm{~mL})$ was added 4-methyl-2-pentanone 5a (20.03 g, $0.19 \mathrm{~mol})$. The resulting mixture was stirred at $75^{\circ} \mathrm{C}$ for $5 \mathrm{~h}$ and left to stand at room temperature overnight. Brine was added and the mixture was extracted with dichloromethane-diethyl ether $(1: 1,3 \mathrm{x}$ $50 \mathrm{~mL}$ ). The combined organic layers were extracted with saturated sodium bicarbonate solution. The aqueous phase was acidified with hydrochloric acid $(10 \mathrm{M})$ and the mixture was extracted with dichloromethane-diethyl ether $(1: 1,2 \times 50 \mathrm{~mL})$. The combined organic extracts were dried $\left(\mathrm{Na}_{2} \mathrm{SO}_{4}\right)$ and concentrated under reduced pressure to give a yellow oil $(18.5 \mathrm{~g}, 60 \%)$. Recrystallization from hexanes yielded alkenoic acid 6a as pale yellow crystals $(1.8 \mathrm{~g}, 10 \%)$. Mp 77-78 ${ }^{\circ} \mathrm{C}$ (lit. $\left.{ }^{21} 76-80{ }^{\circ} \mathrm{C}\right) .{ }^{1} \mathrm{H}$ NMR $\left(300 \mathrm{MHz}, \mathrm{CDCl}_{3}\right) \delta 0.94\left(\mathrm{~m}, 6 \mathrm{H}, \mathrm{CH}_{3}\right), 2.19(\mathrm{~m}, 1 \mathrm{H}, \mathrm{CH})$, $2.53\left(\mathrm{~d}, 2 \mathrm{H}, J=7.2 \mathrm{~Hz}, \mathrm{CH}_{2}\right), 3.69(\mathrm{~s}, 1 \mathrm{H}, \mathrm{OH}), 6.62(\mathrm{~d}, 1 \mathrm{H}, J=15.8 \mathrm{~Hz}, \mathrm{CH}), 7.09$ (d, $1 \mathrm{H}, J=$ $15.8 \mathrm{~Hz}, \mathrm{CH}) .{ }^{13} \mathrm{C} \mathrm{NMR}\left(75.6 \mathrm{MHz}, \mathrm{CDCl}_{3}\right) \delta 22.9\left(\mathrm{CH}_{3}\right), 25.1(\mathrm{CH}), 50.9\left(\mathrm{CH}_{2}\right), 129.9$ $\left(\mathrm{CH}=\underline{\mathrm{CHCO}}{ }_{2} \mathrm{H}\right), 141.8\left(\underline{\mathrm{CH}}=\mathrm{CHCO}_{2} \mathrm{H}\right), 170.8(\mathrm{COOH}), 200.5(\mathrm{CO})$.

(E)-6,6-Dimethyl-4-oxohept-2-enoic acid (6b). This compound was similarly prepared from 4,4-dimethyl-2-pentanone $5 \mathbf{b}(2.50 \mathrm{~g}, 0.02 \mathrm{~mol})$. The crude product was recrystallized from dichloromethane-hexanes to afford alkenoic acid $\mathbf{6 b}$ as white crystals (1.31 g, 40\%). Mp 90$92{ }^{\circ} \mathrm{C}$ (lit. ${ }^{22}$ 91-93 $\left.{ }^{\circ} \mathrm{C}\right) .{ }^{1} \mathrm{H}$ NMR $\left(300 \mathrm{MHz}, \mathrm{CDCl}_{3}\right) \delta 1.03\left(\mathrm{~s}, 9 \mathrm{H}, \mathrm{CH}_{3}\right), 2.53\left(\mathrm{~s}, 2 \mathrm{H}, \mathrm{CH}_{2}\right), 5.99$ $(\mathrm{s}, 1 \mathrm{H}, \mathrm{OH}), 6.58(\mathrm{~d}, 1 \mathrm{H}, J=16.0 \mathrm{~Hz}, \mathrm{CH}), 7.08(\mathrm{~d}, 1 \mathrm{H}, J=16.0 \mathrm{~Hz}, \mathrm{CH}) .{ }^{13} \mathrm{C} \mathrm{NMR}(75.6 \mathrm{MHz}$, $\left.\mathrm{CDCl}_{3}\right) \delta 29.6\left(\mathrm{CH}_{3}\right), 31.6\left(\underline{\mathrm{C}}\left(\mathrm{CH}_{3}\right)_{3}\right), 54.1\left(\mathrm{CH}_{2}\right), 129.1(=\underline{\mathrm{CHCOOH}}), 142.2(\mathrm{CO} \underline{\mathrm{H}}=), 177.1$ $(\mathrm{COOH}), 199.4\left(\mathrm{COCH}_{2}\right)$.

2,3-Dibromo-6-methyl-4-oxoheptanoic acid (7a). To an ice-cold solution of the alkenoic acid 6a $(0.98 \mathrm{~g}, 6.34 \mathrm{mmol})$ in dry dichloromethane $(30 \mathrm{~mL})$ was added dropwise a solution of bromine $(0.29 \mathrm{~mL}, 5.63 \mathrm{mmol})$ in dry dichloromethane $(5 \mathrm{~mL})$. The mixture was stirred at $-78^{\circ} \mathrm{C}$ for $3 \mathrm{~h}$. Saturated aqueous $\mathrm{Na}_{2} \mathrm{~S}_{2} \mathrm{O}_{5}$ was added and the organic phase was washed with water $(2 \times 20 \mathrm{~mL})$, dried $\left(\mathrm{Na}_{2} \mathrm{SO}_{4}\right)$, and concentrated under reduced pressure. The crude product was recrystallized from hexanes to afford dibromo acid 7a as white needles (1.56 g, 78\%). Mp $82{ }^{\circ} \mathrm{C} .{ }^{1} \mathrm{H}$ NMR $(300$ $\left.\mathrm{MHz}, \mathrm{CDCl}_{3}\right) \delta 1.00\left(\mathrm{~m}, 6 \mathrm{H}, \mathrm{CH}_{3}\right), 2.22(\mathrm{~m}, 1 \mathrm{H}, \mathrm{CH}), 2.54\left(\mathrm{~m}, 2 \mathrm{H}, \mathrm{CH}_{2}\right), 4.64(\mathrm{~d}, 1 \mathrm{H}, J=2.6$ $\mathrm{Hz}, \mathrm{CHBr}), 4.72(\mathrm{~d}, 1 \mathrm{H}, J=2.6 \mathrm{~Hz}, \mathrm{CHBr}) .{ }^{13} \mathrm{C} \mathrm{NMR}\left(75.6 \mathrm{MHz}, \mathrm{CDCl}_{3}\right) \delta 22.4\left(\mathrm{CH}_{3}\right), 24.3$ $(\mathrm{CH}), 46.1\left(\underline{\mathrm{C} H B r C O} \mathrm{H}_{2} \mathrm{H}\right), 51.7(\mathrm{CO} \underline{\mathrm{CHBr}}), 171.0\left(\mathrm{CO}_{2} \mathrm{H}\right), 198.6(\mathrm{CO}) . \mathrm{UV}\left(\mathrm{CH}_{3} \mathrm{OH}\right) \lambda_{\max } 206$ $\mathrm{nm}\left(\varepsilon 1997 \mathrm{M}^{-1} \mathrm{~cm}^{-1}\right)$. IR (KBr) v $v_{\max } 3010,2957,2925,2845,1709,1467,1302,1211,1039$, $907 \mathrm{~cm}^{-1}$. 
2,3-Dibromo-6,6-dimethyl-4-oxoheptanoic acid (7b). This compound was similarly prepared from alkenoic acid $\mathbf{6 b}(0.50 \mathrm{~g}, 2.94 \mathrm{mmol})$. The crude product was recrystallized from hexanes to yield the dibromo acid $7 \mathbf{b}$ as white needles $(0.85 \mathrm{~g}, 89 \%)$. Mp 43-47 ${ }^{\circ} \mathrm{C}$. ${ }^{1} \mathrm{H} \mathrm{NMR}(300 \mathrm{MHz}$, $\left.\mathrm{CDCl}_{3}\right) \delta 1.03\left(\mathrm{~m}, 9 \mathrm{H}, \mathrm{CH}_{3}\right), 2.63\left(\mathrm{~m}, 2 \mathrm{H}, \mathrm{CH}_{2}\right), 4.60(\mathrm{~d}, 1 \mathrm{H}, J=1.1 \mathrm{~Hz}, \mathrm{CHBr}), 4.69(\mathrm{~d}, 1 \mathrm{H}, J$ $=1.1 \mathrm{~Hz}, \mathrm{CHBr}) .{ }^{13} \mathrm{C} \mathrm{NMR}\left(75.6 \mathrm{MHz}, \mathrm{CDCl}_{3}\right) \delta 29.4\left(\mathrm{CH}_{3}\right), 31.2\left(\underline{\mathrm{C}}(\mathrm{CH})_{3}\right), 45.4\left(\underline{\mathrm{CHBrCO}} \mathrm{H}_{2}\right)$, $52.9\left(\mathrm{CH}_{2}\right), 53.0(\mathrm{COC} H B r), 172.4\left(\mathrm{CO}_{2} \mathrm{H}\right), 202.0(\mathrm{CO})$. UV $\left(\mathrm{CH}_{3} \mathrm{OH}\right) \lambda_{\max } 202 \mathrm{~nm}\left(\varepsilon 11805 \mathrm{M}^{-1} \mathrm{~cm}^{-1}\right)$, 273 (6260). IR (KBr) $v_{\max }$ 2960, 1712, 1430, 1317, 1290, 1228, 935, $647 \mathrm{~cm}^{-1}$. HRMS (ESI) $\mathrm{m} / \mathrm{z}$ calcd for $\mathrm{C}_{9} \mathrm{H}_{14} \mathrm{Br}_{2} \mathrm{O}_{3} \mathrm{Na}(\mathrm{M}+\mathrm{Na}) 350.9207$, found 350.9201 .

3-Bromo-5-(2-methylpropylidene)furan-2(5H)-one (9a). A mixture of the dibromo acid 7a $(0.37 \mathrm{~g}, 1.17 \mathrm{mmol})$ and phosphorous pentoxide $(0.50 \mathrm{~g}, 1.76 \mathrm{mmol})$ in dry dichloromethane $(15 \mathrm{~mL})$ was refluxed for $1 \mathrm{~h}$. The cooled mixture was filtered through a pad of celite-silicacelite. The filtrate was concentrated under reduced pressure to give 3,4-dibromo-dihydro-5-(2methylpropylidene)furan-2(3H)-one 8 as a yellow oil $(0.21 \mathrm{~g}, 0.70 \mathrm{mmol})$. To the crude oil was added dropwise a solution of DBU $(0.12 \mathrm{~g}, 0.80 \mathrm{mmol})$ in dichloromethane $(3 \mathrm{~mL})$ and the mixture was stirred at $-78{ }^{\circ} \mathrm{C}$ for $1 \mathrm{~h}$. The mixture was acidified with hydrochloric acid (2 M) and extracted with dichloromethane $(3 \times 20 \mathrm{~mL})$. The combined organic layers were dried $\left(\mathrm{Na}_{2} \mathrm{SO}_{4}\right)$ and the solvent was removed under reduced pressure to give a yellow oil. Purification by column chromatography on silica gel (dichloromethane-hexanes, 1:1) afforded furanone 9a as a yellow oil (0.16 g, 63\%). ${ }^{1} \mathrm{H}$ NMR $\left(300 \mathrm{MHz}, \mathrm{CDCl}_{3}\right) \delta 1.08\left(\mathrm{~d}, 6 \mathrm{H}, J=6.8 \mathrm{~Hz}, \mathrm{CH}_{3}\right), 2.96(\mathrm{~m}$, $\left.1 \mathrm{H}, \mathrm{C} \underline{\mathrm{H}}\left(\mathrm{CH}_{3}\right)_{2}\right), 5.19(\mathrm{~d}, 1 \mathrm{H}, J=9.8 \mathrm{~Hz},=\mathrm{CH}), 7.39(\mathrm{~s}, 1 \mathrm{H}, \mathrm{H} 4) .{ }^{13} \mathrm{C} \mathrm{NMR}\left(75.6 \mathrm{MHz}, \mathrm{CDCl}_{3}\right) \delta$ $22.4(\mathrm{CH}), 26.2\left(\mathrm{CH}_{3}\right), 111.7\left(=\underline{\mathrm{CHCH}}\left(\mathrm{CH}_{3}\right)_{2}\right), 124.8(\mathrm{C} 3), 141.5(\mathrm{C} 4), 146.2$ (C5), $166.7(\mathrm{C} 2)$. $\mathrm{UV}\left(\mathrm{CH}_{3} \mathrm{OH}\right) \lambda_{\max } 284 \mathrm{~nm}\left(\varepsilon 15659 \mathrm{M}^{-1} \mathrm{~cm}^{-1}\right)$. IR (KBr) v $v_{\max } 3083,2964,1781,1466,1299$, 1220, 1095, 991, 904, $754 \mathrm{~cm}^{-1}$. MS (MALDI) m/z $217\left([\mathrm{M}+\mathrm{H}]^{+}\left({ }^{79} \mathrm{Br}\right), 100 \%\right), 219\left([\mathrm{M}+\mathrm{H}]^{+}\right.$ $\left.\left({ }^{81} \mathrm{Br}\right), 86 \%\right)$.

3-Bromo-5-(2,2-dimethylpropylidene)furan-2(5H)-one (9b). This compound was similarly prepared from dibromo acid $\mathbf{7 b}(3.6 \mathrm{~g}, 10.9 \mathrm{mmol})$. The desired furanone $\mathbf{9 b}$ was obtained as a yellow solid (1.7 g, $7.4 \mathrm{mmol}, 75 \%)$. Mp 56-58 ${ }^{\circ} \mathrm{C} .{ }^{1} \mathrm{H}$ NMR $\left(300 \mathrm{MHz}, \mathrm{CDCl}_{3}\right) \delta 1.24(\mathrm{~m}, 9 \mathrm{H}$, $\left.\mathrm{CH}_{3}\right), 5.26(\mathrm{~s}, 1 \mathrm{H},=\mathrm{CH}), 7.35(\mathrm{~s}, 1 \mathrm{H}, \mathrm{H} 4) .{ }^{13} \mathrm{C} \mathrm{NMR}\left(75.6 \mathrm{MHz}, \mathrm{CDCl}_{3}\right) \delta 30.7\left(\mathrm{CH}_{3}\right), 33.6$ $\left(\underline{\mathrm{C}}\left(\mathrm{CH}_{3}\right)_{3}\right), 110.6\left(=\underline{\mathrm{CHC}}\left(\mathrm{CH}_{3}\right)_{3}\right), 128.0(\mathrm{C} 3), 142.9(\mathrm{C} 4), 146.1(\mathrm{C} 5), 165.6(\mathrm{C} 2) . \mathrm{UV}\left(\mathrm{CH}_{3} \mathrm{OH}\right)$ $\lambda_{\max } 285 \mathrm{~nm}\left(\varepsilon 29549 \mathrm{M}^{-1} \mathrm{~cm}^{-1}\right) . \mathrm{IR}(\mathrm{KBr}) v_{\max } 3309,2967,1748,1560,1339,1244,1220,1002$, 960, $900 \mathrm{~cm}^{-1}$. Anal. Calcd for $\mathrm{C}_{9} \mathrm{H}_{11} \mathrm{BrO}_{2}$ : C, 46.78; H, 4.80. Found: C, 46.76; H, 4.78

4-Bromo-5-(2-methylpropylidene)furan-2(5H)-one (11a). The dibromo acid 7a (0.704 g, $2.22 \mathrm{mmol})$ was dissolved in sulfuric acid $(18 \mathrm{M}, 5 \mathrm{~mL})$ and the solution was heated at $100{ }^{\circ} \mathrm{C}$ for $10 \mathrm{~min}$. The cooled mixture was poured into ice-water $(50 \mathrm{~mL})$ and extracted with dichloromethane-diethyl ether $(1: 1,3 \times 50 \mathrm{~mL})$. The organic phase was washed with saturated sodium bicarbonate solution $(30 \mathrm{~mL})$, dried $\left(\mathrm{Na}_{2} \mathrm{SO}_{4}\right)$, and concentrated under reduced pressure. The crude product was purified by column chromatography on silica gel (dichloromethanehexanes, 2:3) to yield furanone 11a as a yellow oil $(0.26 \mathrm{~g}, 54 \%) .{ }^{1} \mathrm{H} \mathrm{NMR}\left(300 \mathrm{MHz}, \mathrm{CDCl}_{3}\right) \delta$ $1.10\left(\mathrm{~m}, 6 \mathrm{H}, \mathrm{CH}_{3}\right), 2.97\left(\mathrm{~m}, 1 \mathrm{H}, \mathrm{C} \underline{\mathrm{H}}\left(\mathrm{CH}_{3}\right)_{2}\right), 5.44(\mathrm{~d}, 1 \mathrm{H}, J=9.8 \mathrm{~Hz},=\mathrm{CH}), 6.32(\mathrm{~s}, 1 \mathrm{H}, \mathrm{H} 3)$. ${ }^{13} \mathrm{C}$ NMR $\left(75.6 \mathrm{MHz}, \mathrm{CDCl}_{3}\right) \delta 22.2(\mathrm{CH}), 26.5\left(\mathrm{CH}_{3}\right), 119.6\left(=\underline{\mathrm{CHCH}}\left(\mathrm{CH}_{3}\right)_{2}\right), 123.7(\mathrm{C} 3)$, 
137.1 (C4), 146.5 (C5), 166.9 (C2). UV ( $\left(\mathrm{CH}_{3} \mathrm{OH}\right) \lambda_{\max } 245 \mathrm{~nm}\left(\varepsilon 37150 \mathrm{M}^{-1} \mathrm{~cm}^{-1}\right), 335$ (27869). IR (KBr) $v_{\max } 3023,2969,1770,1557,1215,1108,983,753 \mathrm{~cm}^{-1}$. HRMS (ESI) $\mathrm{m} / \mathrm{z}$ calcd for $\mathrm{C}_{8} \mathrm{H}_{9} \mathrm{BrO}_{2} \mathrm{Na}(\mathrm{M}+\mathrm{Na})^{+} 238.9678$, found 238.9672 .

4-Bromo-5-(2,2-dimethylpropylidene)furan-2(5H)-one (11b). This compound was similarly prepared from 2,3-dibromo-6,6-dimethyl-4-oxoheptanoic acid $7 \mathbf{b}(2.0 \mathrm{~g}, 6.1 \mathrm{mmol})$. The desired furanone 11b was obtained as a yellow oil $(0.35 \mathrm{~g}, 1.5 \mathrm{mmol}, 25 \%) .{ }^{1} \mathrm{H} \mathrm{NMR}\left(300 \mathrm{MHz}, \mathrm{CDCl}_{3}\right)$ $\delta 1.25\left(\mathrm{~s}, 9 \mathrm{H}, \mathrm{CH}_{3}\right), 5.54(\mathrm{~s}, 1 \mathrm{H},=\mathrm{CH}), 6.28(\mathrm{~s}, 1 \mathrm{H}, \mathrm{H} 3) .{ }^{13} \mathrm{C} \mathrm{NMR}\left(75.6 \mathrm{MHz}, \mathrm{CDCl}_{3}\right) \delta 29.9$ $\left(\mathrm{CH}_{3}\right), 33.2\left(\underline{\mathrm{C}}\left(\mathrm{CH}_{3}\right)_{3}\right), 118.7\left(=\underline{\mathrm{CHC}}\left(\mathrm{CH}_{3}\right)_{3}\right), 126.2(\mathrm{C} 3), 138.4(\mathrm{C} 4), 146.1(\mathrm{C} 5), 167.1(\mathrm{C} 2)$. $\mathrm{UV}\left(\mathrm{CH}_{3} \mathrm{OH}\right) \lambda_{\max } 202 \mathrm{~nm}\left(\varepsilon 3067 \mathrm{M}^{-1} \mathrm{~cm}^{-1}\right), 279$ (14830). IR $v_{\max } 3140,2963,2870,1770$, 1560, 1267, 1224, 1109, 985, 941, 908, $804 \mathrm{~cm}^{-1}$. HRMS (ESI) $\mathrm{m} / \mathrm{z}$ calcd for $\mathrm{C}_{9} \mathrm{H}_{11} \mathrm{BrO}_{2} \mathrm{Na}$ $(\mathrm{M}+\mathrm{Na})^{+} 252.9840$, found 252.9847 .

\section{General procedure for Suzuki-Miyaura coupling reactions}

To a solution of the furanone $\mathbf{9}$ or $11(231 \mathrm{mg}, 1.00 \mathrm{mmol})$ in toluene $(10 \mathrm{~mL})$ and water $(10 \mathrm{~mL})$ was added boronic acid $12(1.20 \mathrm{mmol}), \mathrm{CsF}(456 \mathrm{mg}, 3.00 \mathrm{mmol}), \mathrm{Bu}_{4} \mathrm{NI}(18 \mathrm{mg}, 0.05 \mathrm{mmol})$ and $\mathrm{Pd}\left(\mathrm{PPh}_{3}\right) \mathrm{Cl}_{2}(35 \mathrm{mg}, 0.05 \mathrm{mmol})$. The mixture was refluxed for 36 hours under a nitrogen atmosphere. Brine $(40 \mathrm{~mL})$ was added and the mixture was extracted with ethyl acetate $(3 \mathrm{x}$ $50 \mathrm{~mL})$. The combined organic layers were dried $\left(\mathrm{Na}_{2} \mathrm{SO}_{4}\right)$ and the solvent was removed under reduced pressure. The crude product was purified by flash chromatography on silica gel and recrystallized from dichloromethane-hexanes.

5-(2,2-Dimethylpropylidene)-3-phenylfuran-2(5H)-one (13a). Yellow crystals (89\%). Mp 59$62{ }^{\circ} \mathrm{C} .{ }^{1} \mathrm{H}$ NMR $\left(300 \mathrm{MHz}, \mathrm{CDCl}_{3}\right) \delta 1.29\left(\mathrm{~s}, 9 \mathrm{H}, \mathrm{CH}_{3}\right), 5.27(\mathrm{~s}, 1 \mathrm{H},=\mathrm{CH}), 7.38-7.42(\mathrm{~m}, 4 \mathrm{H}$, $\mathrm{ArH}), 7.39(\mathrm{~s}, 1 \mathrm{H}, \mathrm{H} 4), 7.85-7.86(\mathrm{~m}, 1 \mathrm{H}, \mathrm{ArH}) .{ }^{13} \mathrm{C} \mathrm{NMR}\left(75.6 \mathrm{MHz}, \mathrm{CDCl}_{3}\right) \delta 30.3\left(\mathrm{CH}_{3}\right)$, $33.3\left(\underline{\mathrm{C}}\left(\mathrm{CH}_{3}\right)_{3}\right), 126.6\left(=\underline{\mathrm{CHC}}\left(\mathrm{CH}_{3}\right)_{3}\right), 126.9(\mathrm{Ar}), 128.4(\mathrm{Ar}), 128.6(\mathrm{Ar}), 129.2(\mathrm{Ar}), 129.5(\mathrm{C} 3)$, 136.8 (C4), 146.2 (C5), $168.8(\mathrm{C} 2)$. UV ( $\left.\mathrm{CH}_{3} \mathrm{OH}\right) \lambda_{\max } 203 \mathrm{~nm}\left(\varepsilon 28185 \mathrm{M}^{-1} \mathrm{~cm}^{-1}\right), 232(20212)$, 312 (49152). IR (KBr) v $v_{\max } 3100,2957,1750,1447,1295,1096,962,906,787,746 \mathrm{~cm}^{-1}$. HRMS (ESI) $\mathrm{m} / \mathrm{z}$ calcd for $\mathrm{C}_{15} \mathrm{H}_{17} \mathrm{O}_{2}(\mathrm{M}+\mathrm{H})^{+}$229.1229, found 229.1236. Anal. Calcd for $\mathrm{C}_{15} \mathrm{H}_{16} \mathrm{O}_{2}$ : C, 78.92; H, 7.06. Found: C, 78.62; H, 7.22.

3-(4-Fluorophenyl)-5-(2,2-dimethylpropylidene)furan-2(5H)-one (13b). White needles (39\%). Mp 129-131 ${ }^{\circ} \mathrm{C} .{ }^{1} \mathrm{H}$ NMR $\left(300 \mathrm{MHz}, \mathrm{CDCl}_{3}\right) \delta 1.28\left(\mathrm{~s}, 9 \mathrm{H}, \mathrm{CH}_{3}\right), 5.27(\mathrm{~s}, 1 \mathrm{H},=\mathrm{CH}), 7.10(\mathrm{~m}$, $2 \mathrm{H}, \mathrm{ArH}), 7.34(\mathrm{~s}, 1 \mathrm{H}, 4 \mathrm{H}), 7.85-7.90(\mathrm{~m}, 2 \mathrm{H}, \mathrm{ArH}) .{ }^{13} \mathrm{C} \mathrm{NMR}\left(75.6 \mathrm{MHz}, \mathrm{CDCl}_{3}\right) \delta 30.2\left(\mathrm{CH}_{3}\right)$, $33.3\left(\underline{\mathrm{C}}\left(\mathrm{CH}_{3}\right)_{3}\right), 115.6(\mathrm{Ar}), 115.9\left(=\underline{\mathrm{C}} \mathrm{HC}\left(\mathrm{CH}_{3}\right)_{3}\right), 128.8(\mathrm{Ar}), 128.9(\mathrm{C} 3), 136.4(\mathrm{C} 4), 146.0$ (C5), 161.5 (Ar), 164.8 (Ar), $168.7(\mathrm{C} 2)$. UV $\left(\mathrm{CH}_{3} \mathrm{OH}\right) \lambda_{\max } 203 \mathrm{~nm}\left(\varepsilon 23234 \mathrm{M}^{-1} \mathrm{~cm}^{-1}\right), 232$ (16662), 310 (47453). IR (KBr) $v_{\max }$ 3085, 2961, 1754, 1507, 1344, 1295, 1235, 1165, 1108, 965 , $835,528 \mathrm{~cm}^{-1}$. HRMS (ESI) $\mathrm{m} / z$ calcd for $\mathrm{C}_{15} \mathrm{H}_{16} \mathrm{FO}_{2}(\mathrm{M}+\mathrm{H})^{+} 247.1134$, found 247.1120.

3-(3-Fluorophenyl)-5-(2,2-dimethylpropylidene)furan-2(5H)-one (13c). White needles (66\%). Mp 62-65 ${ }^{\circ} \mathrm{C} .{ }^{1} \mathrm{H}$ NMR $\left(300 \mathrm{MHz}, \mathrm{CDCl}_{3}\right) \delta 1.28\left(\mathrm{~s}, 9 \mathrm{H}, \mathrm{CH}_{3}\right), 5.31(\mathrm{~s}, 1 \mathrm{H},=\mathrm{CH}), 7.05-7.06(\mathrm{~m}$, $1 \mathrm{H}, \mathrm{ArH}), 7.36-7.39(\mathrm{~m}, 1 \mathrm{H}, \mathrm{ArH}), 7.41(\mathrm{~s}, 1 \mathrm{H}, \mathrm{H} 4), 7.60-7.61(\mathrm{~m}, 2 \mathrm{H}, \mathrm{ArH}) .{ }^{13} \mathrm{C}$ NMR $(75.6$ $\left.\mathrm{MHz}, \mathrm{CDCl}_{3}\right) \delta 30.2\left(\mathrm{CH}_{3}\right), 33.4\left(\underline{\mathrm{C}}\left(\mathrm{CH}_{3}\right)_{3}\right), 114.0(\mathrm{Ar}), 115.9\left(=\underline{\mathrm{CHC}}\left(\mathrm{CH}_{3}\right)_{3}\right), 122.6(\mathrm{Ar}), 127.7$ (Ar), 130.1 (Ar), 131.4 (C3), 137.6(C4), 146.0 (C5), 161.2 (Ar), 164.4 (Ar), 168.3 (C2) . UV 
$\left(\mathrm{CH}_{3} \mathrm{OH}\right) \lambda_{\max } 204 \mathrm{~nm}\left(\varepsilon 40655 \mathrm{M}^{-1} \mathrm{~cm}^{-1}\right), 230$ (21748), 309 (66060). IR (KBr) $v_{\max } 3118,2963$, 1748, 1652, 1489, 1345, 1280, 1224, 1090, 966, 904, $763 \mathrm{~cm}^{-1}$. HRMS (ESI) $\mathrm{m} / \mathrm{z}$ calcd for $\mathrm{C}_{15} \mathrm{H}_{16} \mathrm{FO}_{2}(\mathrm{M}+\mathrm{H})^{+}$247.1134, found 247.1123. Anal. Calcd for $\mathrm{C}_{15} \mathrm{H}_{15} \mathrm{FO}_{2}$ : C, 73.15; H, 6.14. Found: C, 73.08; H, 6.24.

3-(2-Fluorophenyl)-5-(2,2-dimethylpropylidene)furan-2(5H)-one (13d). White needles (74\%). Mp 91-94 ${ }^{\circ} \mathrm{C} .{ }^{1} \mathrm{H}$ NMR $\left(300 \mathrm{MHz}, \mathrm{CDCl}_{3}\right) \delta 1.29\left(\mathrm{~s}, 9 \mathrm{H}, \mathrm{CH}_{3}\right), 5.34(\mathrm{~s}, 1 \mathrm{H},=\mathrm{CH}), 7.09-7.64(\mathrm{~m}$, $3 \mathrm{H}, \mathrm{ArH}), 7.65(\mathrm{~s}, 1 \mathrm{H}, \mathrm{H} 4), 8.28-8.29(\mathrm{~m}, 1 \mathrm{H}, \mathrm{ArH}) .{ }^{13} \mathrm{C} \mathrm{NMR}\left(75.6 \mathrm{MHz}, \mathrm{CDCl}_{3}\right) \delta 30.2\left(\mathrm{CH}_{3}\right)$, $33.4\left(\underline{\mathrm{C}}\left(\mathrm{CH}_{3}\right)_{3}\right), 115.8\left(=\underline{\mathrm{CHC}}\left(\mathrm{CH}_{3}\right)_{3}\right), 117.9$ (Ar), 124.3 (Ar), 127.8 (Ar), 129.5 (Ar), $130.4(\mathrm{C} 3)$, 140.1(C4), 146.3 (C5), 161.2 (Ar), 164.4 (Ar), 168.9 (C2). UV ( $\left.\mathrm{CH}_{3} \mathrm{OH}\right) \lambda_{\max } 202 \mathrm{~nm}(\varepsilon 23208$ $\mathrm{M}^{-1} \mathrm{~cm}^{-1}$ ), 230 (7258), 310 (23759). IR (KBr) v $v_{\max }$ 3097, 2960, 1752, 1440, 1330, 1193, 1097 , 967, 864, $788 \mathrm{~cm}^{-1}$. HRMS (ESI) $\mathrm{m} / \mathrm{z}$ calcd for $\mathrm{C}_{15} \mathrm{H}_{16} \mathrm{FO}_{2}(\mathrm{M}+\mathrm{H})^{+}$247.1134, found 247.1107. Anal. Calcd for $\mathrm{C}_{15} \mathrm{H}_{15} \mathrm{FO}_{2}$ : C, 73.15; H, 6.14. Found: C, 73.03; H, 6.18.

3-(2,4-Difluorophenyl)-5-(2,2-dimethylpropylidene)furan-2(5H)-one (13e). Yellow crystals (53\%). Mp 156-157 ${ }^{\circ} \mathrm{C} .{ }^{1} \mathrm{H}$ NMR $\left(300 \mathrm{MHz}, \mathrm{CDCl}_{3}\right) \delta 1.28\left(\mathrm{~s}, 9 \mathrm{H}, \mathrm{CH}_{3}\right), 5.34(\mathrm{~s}, 1 \mathrm{H},=\mathrm{CH})$, 6.86-7.00 (m, 2H, ArH), 7.60 (s, 1H, H4), 8.30-8.31 (m, 1H, ArH). ${ }^{13} \mathrm{C}$ NMR $\left(75.6 \mathrm{MHz}, \mathrm{CDCl}_{3}\right)$ $\delta 30.2\left(\mathrm{CH}_{3}\right), 33.4\left(\underline{\mathrm{C}}\left(\mathrm{CH}_{3}\right)_{3}\right), 104.3(\mathrm{Ar}), 114.3(\mathrm{Ar}), 111.7\left(=\underline{\mathrm{CHC}}\left(\mathrm{CH}_{3}\right)_{3}\right), 128.0(\mathrm{Ar}), 130.6$ (Ar), 121.0 (C3), 140.4 (C4), 146.2 (C5), 162.8 (Ar), 164.4 (Ar), 168.8 (C2). UV ( $\left.\mathrm{CH}_{3} \mathrm{OH}\right) \lambda_{\max }$ $202 \mathrm{~nm}\left(\varepsilon 16678 \mathrm{M}^{-1} \mathrm{~cm}^{-1}\right), 228$ (8442), 306 (30655). IR (KBr) v $v_{\max }$ 3130, 2960, 1751, 1586, 1501, 1422, 1282, 1141, 1090, 913, 840, 813, 772, 712, $590 \mathrm{~cm}^{-1}$. HRMS (ESI) $\mathrm{m} / \mathrm{z}$ calcd for $\mathrm{C}_{15} \mathrm{H}_{15} \mathrm{~F}_{2} \mathrm{O}_{2}(\mathrm{M}+\mathrm{H})^{+}$265.1040, found 265.1021. Anal. Calcd for $\mathrm{C}_{15} \mathrm{H}_{14} \mathrm{~F}_{2} \mathrm{O}_{2}: \mathrm{C}, 68.17 ; \mathrm{H}, 5.34$. Found: C, 68.10; H, 5.36.

3-(4-(Trifluoromethyl)phenyl)-5-(2,2-dimethylpropylidene)furan-2(5H)-one (13f). Yellow crystals (61\%). Mp 120-122 ${ }^{\circ} \mathrm{C} .{ }^{1} \mathrm{H}$ NMR $\left(300 \mathrm{MHz}, \mathrm{CDCl}_{3}\right) \delta 1.29\left(\mathrm{~s}, 9 \mathrm{H}, \mathrm{CH}_{3}\right), 5.36(\mathrm{~s}, 1 \mathrm{H}$, $=\mathrm{CH}), 7.50(\mathrm{~s}, 1 \mathrm{H}, \mathrm{H} 4), 7.65-7.68(\mathrm{~d}, J=8.1 \mathrm{~Hz}, 2 \mathrm{H}, \mathrm{ArH}), 7.97-7.98(\mathrm{~d}, J=8.1 \mathrm{~Hz}, 2 \mathrm{H}, \mathrm{ArH})$. ${ }^{13} \mathrm{C}$ NMR $\left(75.6 \mathrm{MHz}, \mathrm{CDCl}_{3}\right) \delta 30.2\left(\mathrm{CH}_{3}\right), 33.5\left(\underline{\mathrm{C}}\left(\mathrm{CH}_{3}\right)_{3}\right), 122.0(\mathrm{Ar}), 125.6\left(=\underline{\mathrm{CHC}}\left(\mathrm{CH}_{3}\right)_{3}\right)$, 125.6 (Ar), 127.1 (Ar), $129.5\left(\mathrm{CF}_{3}\right), 130.6$ (C3), 132.9 (Ar), 138.5 (C4), 146.0 (C5), 168.3 (C2). $\mathrm{UV}\left(\mathrm{CH}_{3} \mathrm{OH}\right) \lambda_{\max } 202 \mathrm{~nm}\left(\varepsilon 29392 \mathrm{M}^{-1} \mathrm{~cm}^{-1}\right), 229$ (13986), 308 (53744). IR (KBr) v $v_{\max } 3078$, 2962, 1754, 1332, 1160, 1129, 1071, 967, $720 \mathrm{~cm}^{-1}$. HRMS (ESI) $\mathrm{m} / \mathrm{z}$ calcd for $\mathrm{C}_{16} \mathrm{H}_{16} \mathrm{~F}_{3} \mathrm{O}_{2}$ $(\mathrm{M}+\mathrm{H})^{+}$297.1102, found 297.1060. Anal. Calcd for $\mathrm{C}_{16} \mathrm{H}_{15} \mathrm{~F}_{3} \mathrm{O}_{2}: \mathrm{C}, 64.86 ; \mathrm{H}, 5.10$. Found: C, $65.00 ; \mathrm{H}, 5.14$.

3-(3-(Trifluoromethyl)phenyl)-5-(2,2-dimethylpropylidene)furan-2(5H)-one (13g). Yellow crystals (70\%). Mp 118-122 ${ }^{\circ} \mathrm{C} .{ }^{1} \mathrm{H}$ NMR $\left(300 \mathrm{MHz}, \mathrm{CDCl}_{3}\right) \delta 1.29\left(\mathrm{~s}, 9 \mathrm{H}, \mathrm{CH}_{3}\right), 5.35(\mathrm{~s}, 1 \mathrm{H}$, $=\mathrm{CH}), 7.49(\mathrm{~s}, 1 \mathrm{H}, \mathrm{H} 4), 7.52-7.64(\mathrm{~m}, 2 \mathrm{H}, \mathrm{ArH}), 8.09-8.13(\mathrm{~m}, 2 \mathrm{H}, \mathrm{ArH}) .{ }^{13} \mathrm{C} \mathrm{NMR}(75.6 \mathrm{MHz}$, $\left.\mathrm{CDCl}_{3}\right) \delta 30.2\left(\mathrm{CH}_{3}\right), 33.5\left(\underline{\mathrm{C}}\left(\mathrm{CH}_{3}\right)_{3}\right), 122.0(\mathrm{Ar}), 123.6(\mathrm{Ar}), 123.7(\mathrm{Ar}), 125.7\left(=\underline{\mathrm{CHC}}\left(\mathrm{CH}_{3}\right)_{3}\right)$, 125.7 (Ar), 126.9 (Ar), 128.2 (Ar), $130.2\left(\mathrm{CF}_{3}\right), 130.9$ (C3), 138.0 (C4), 145.9 (C5), 168.3 (C2). $\mathrm{UV}\left(\mathrm{CH}_{3} \mathrm{OH}\right) \lambda_{\max } 202 \mathrm{~nm}\left(\varepsilon 20999 \mathrm{M}^{-1} \mathrm{~cm}^{-1}\right), 231$ (13184), 309 (42387). IR (KBr) v $v_{\max } 2965$, $1772,1655,1431,1350,1281,1164,1121,1097,882,808,695,677 \mathrm{~cm}^{-1}$. HRMS (ESI) m/z calcd for $\mathrm{C}_{16} \mathrm{H}_{16} \mathrm{~F}_{3} \mathrm{O}_{2}(\mathrm{M}+\mathrm{H})^{+}$297.1102, found 297.1073. Anal. Calcd for $\mathrm{C}_{16} \mathrm{H}_{15} \mathrm{~F}_{3} \mathrm{O}_{2}: \mathrm{C}, 64.86$; H, 5.10. Found: C, 65.15; H, 5.18. 
3-(4-Cyanophenyl)-5-(2,2-dimethylpropylidene)furan-2(5H)-one (13h). Yellow crystals (45\%). Mp 150-153 ${ }^{\circ} \mathrm{C} .{ }^{1} \mathrm{H}$ NMR $\left(300 \mathrm{MHz}, \mathrm{CDCl}_{3}\right) \delta 1.29\left(\mathrm{~s}, 9 \mathrm{H}, \mathrm{CH}_{3}\right), 5.40(\mathrm{~s}, 1 \mathrm{H},=\mathrm{CH})$, $7.52(\mathrm{~s}, 1 \mathrm{H}, 4 \mathrm{H}), 7.71-7.86(\mathrm{~d}, J=8.7 \mathrm{~Hz}, 2 \mathrm{H}, \mathrm{ArH}), 7.99-8.01(\mathrm{~d}, J=8.7 \mathrm{~Hz}, 2 \mathrm{H}, \mathrm{ArH}) .{ }^{13} \mathrm{C}$ NMR (75.6 MHz, $\left.\mathrm{CDCl}_{3}\right) \delta 30.1\left(\mathrm{CH}_{3}\right), 33.6\left(\underline{\mathrm{C}}\left(\mathrm{CH}_{3}\right)_{3}\right), 112.5(\mathrm{Ar}), 118.4(\mathrm{CN}), 126.4(\mathrm{Ar})$, $127.6\left(=\underline{\mathrm{CHC}}\left(\mathrm{CH}_{3}\right)_{3}\right), 129.4(\mathrm{Ar}), 132.4$ (Ar), 133.8 (C3), 139.1 (C4), 145.9 (C5), 168.0 (C2). $\mathrm{UV}\left(\mathrm{CH}_{3} \mathrm{OH}\right) \lambda_{\max } 203 \mathrm{~nm}\left(\varepsilon 22227 \mathrm{M}^{-1} \mathrm{~cm}^{-1}\right), 317$ (51758). IR (KBr) v $v_{\max }$ 3084, 2970, 2221, 1784, 1766, 1606, 1296, 1090, 956, 902, 846, $553 \mathrm{~cm}^{-1}$. HRMS (ESI) $\mathrm{m} / \mathrm{z}$ calcd for $\mathrm{C}_{16} \mathrm{H}_{15} \mathrm{NO}_{2} \mathrm{Na}(\mathrm{M}+\mathrm{Na}) 276.1000$, found 276.0996. Anal. Calcd for $\mathrm{C}_{16} \mathrm{H}_{15} \mathrm{NO}_{2}$ : C, 75.85; H, 5.97; N, 5.53. Found: C, 75.53; H, 6.28; N, 5.27.

3-(1H-Indol-5-yl)-5-(2,2-dimethylpropylidene)furan-2(5H)-one (13i). Yellow crystals (21\%). Mp 168-171 ${ }^{\circ} \mathrm{C} .{ }^{1} \mathrm{H}$ NMR $\left(300 \mathrm{MHz}, \mathrm{CDCl}_{3}\right) \delta 1.29\left(\mathrm{~s}, 9 \mathrm{H}, \mathrm{CH}_{3}\right), 5.20(\mathrm{~s}, 1 \mathrm{H},=\mathrm{CH}), 6.60-6.61$ (m, 1H, ArH), 7.22-7.23 (m, 1H, ArH), 7.34 (s, 1H, 4H), 7.41 (d, 1H, J = 8.6 Hz, ArH), 7.64 (d, $1 \mathrm{H}, J=8.6 \mathrm{~Hz}, \mathrm{ArH}), 8.31(\mathrm{~s}, 1 \mathrm{H}, \mathrm{ArH}) .{ }^{13} \mathrm{C} \mathrm{NMR}\left(75.6 \mathrm{MHz}, \mathrm{CDCl}_{3}\right) \delta 30.4\left(\mathrm{CH}_{3}\right), 33.2$ $\left(\underline{\mathrm{C}}\left(\mathrm{CH}_{3}\right)_{3}\right), 103.5(\mathrm{Ar}), 110.3(\mathrm{Ar}), 111.3\left(=\underline{\mathrm{CHC}}\left(\mathrm{CH}_{3}\right)_{3}\right), 121.0(\mathrm{Ar}), 121.5$ (Ar), 124.0 (Ar), 125.0 (Ar), 127.5 (Ar), 128.0 (Ar), 134.7 (C4), 136.1 (C3), 146.3 (C5), 168.9 (C2). UV (CH $\mathrm{CH}_{3} \mathrm{OH}$ $\lambda_{\max } 202 \mathrm{~nm}$ (ع $23208 \mathrm{M}^{-1} \mathrm{~cm}^{-1}$ ), 230 (7258), 310 (23759). IR (KBr) $v_{\max }$ 3097, 2960, 1752, 1440, 1330, 1193, 1097, 967, 864, $788 \mathrm{~cm}^{-1}$. HRMS (ESI) $\mathrm{m} / \mathrm{z}$ calcd for $\mathrm{C}_{17} \mathrm{H}_{18} \mathrm{NO}_{2}(\mathrm{M}+\mathrm{H})^{+} 268.1338$, found 268.1312 .

4-(4-Fluorophenyl)-5-(2,2-dimethylpropylidene)furan-2(5H)-one (14a). White solid (70\%). Mp 59-62 ${ }^{\circ} \mathrm{C} .{ }^{1} \mathrm{H}$ NMR $\left(300 \mathrm{MHz}, \mathrm{CDCl}_{3}\right) \delta 1.27\left(\mathrm{~s}, 9 \mathrm{H}, \mathrm{CH}_{3}\right), 5.32(\mathrm{~s}, 1 \mathrm{H},=\mathrm{CH}), 6.08(\mathrm{~s}, 1 \mathrm{H}$, $3 \mathrm{H})$, 7.14-7.20 (m, 2H, ArH), 7.38-7.40 (m, 2H, ArH). $\left.{ }^{13} \mathrm{C} \mathrm{NMR} \mathrm{(75.6} \mathrm{MHz,} \mathrm{CDCl}_{3}\right) \delta 30.3$ $\left(\mathrm{CH}_{3}\right), 33.3\left(\underline{\mathrm{C}}\left(\mathrm{CH}_{3}\right)_{3}\right), 114.4\left(=\underline{\mathrm{CHC}}\left(\mathrm{CH}_{3}\right)_{3}\right), 115.9(\mathrm{Ar}), 126.6(\mathrm{Ar}), 130.3(\mathrm{C} 3), 147.2(\mathrm{C} 5)$, 157.2 (C4), 162.1(Ar), 165.5(Ar), $168.5(\mathrm{C} 2)$. UV (CH $\left.\mathrm{CH}_{3} \mathrm{OH}\right) \lambda_{\max } 201 \mathrm{~nm}\left(\varepsilon 7937 \mathrm{M}^{-1} \mathrm{~cm}^{-1}\right), 294$ (5866). IR (KBr) v $v_{\max } 2963,2869,1770,1613,1505,1327,1231,1161,1083,977,833,799,584$ $\mathrm{cm}^{-1}$. HRMS (ESI) $\mathrm{m} / \mathrm{z}$ calcd for $\mathrm{C}_{15} \mathrm{H}_{15} \mathrm{FO}_{2} \mathrm{Na}(\mathrm{M}+\mathrm{Na})^{+} 269.0954$, found 269.0955.

4-(3-Fluorophenyl)-5-(2,2-dimethylpropylidene)furan-2(5H)-one (14b). Yellow solid (25\%). Mp 42-44 ${ }^{\circ} \mathrm{C} .{ }^{1} \mathrm{H}$ NMR $\left(300 \mathrm{MHz}, \mathrm{CDCl}_{3}\right) \delta 1.27\left(\mathrm{~s}, 9 \mathrm{H}, \mathrm{CH}_{3}\right), 5.35(\mathrm{~s}, 1 \mathrm{H},=\mathrm{CH}), 6.11(\mathrm{~s}, 1 \mathrm{H}$, $3 \mathrm{H}), 7.10-7.13(\mathrm{~m}, 1 \mathrm{H}, \mathrm{ArH}), 7.17-7.22(\mathrm{~m}, 2 \mathrm{H}, \mathrm{ArH}), 7.44-7.47$ (m, 1H, ArH). ${ }^{13} \mathrm{C}$ NMR $(75.6$ $\left.\mathrm{MHz}, \mathrm{CDCl}_{3}\right) \delta 30.2\left(\mathrm{CH}_{3}\right), 33.3\left(\underline{\mathrm{C}}\left(\mathrm{CH}_{3}\right)_{3}\right), 115.0\left(=\underline{\mathrm{CHC}}\left(\mathrm{CH}_{3}\right)_{3}\right), 115.7(\mathrm{Ar}), 117.2(\mathrm{Ar}), 124.2$ (Ar), 126.7 (Ar), 130.5 (C3), 147.0 (C5), 156.9 (C4), 160.9(Ar), 164.1(Ar), 168.4 (C2). UV $\left(\mathrm{CH}_{3} \mathrm{OH}\right) \lambda_{\max } 202 \mathrm{~nm}\left(\varepsilon 19408 \mathrm{M}^{-1} \mathrm{~cm}^{-1}\right), 239$ (9926), 334 (19162). IR (KBr) $v_{\max } 3100,1771$, $1576,1432,1272,1197,1182,930,874,796,682 \mathrm{~cm}^{-1}$. HRMS (ESI) $\mathrm{m} / \mathrm{z}$ calcd for $\mathrm{C}_{15} \mathrm{H}_{15} \mathrm{FO}_{2} \mathrm{Na}$ $(\mathrm{M}+\mathrm{Na})^{+} 269.0954$, found 269.0948 .

4-(2-Fluorophenyl)-5-(2,2-dimethylpropylidene)furan-2(5H)-one (14c). Yellow solid (25\%). Mp 39-41 ${ }^{\circ} \mathrm{C} .{ }^{1} \mathrm{H}$ NMR $\left(300 \mathrm{MHz}, \mathrm{CDCl}_{3}\right) \delta 1.26\left(\mathrm{~s}, 9 \mathrm{H}, \mathrm{CH}_{3}\right), 5.23(\mathrm{~s}, 1 \mathrm{H},=\mathrm{CH}), 6.19(\mathrm{~s}, 1 \mathrm{H}$, $3 \mathrm{H}), 7.17-7.23(\mathrm{~m}, 1 \mathrm{H}, \mathrm{ArH}), 7.31-7.34$ (m, 2H, ArH), 7.43-7.50 (m, 1H, ArH). ${ }^{13} \mathrm{C}$ NMR (75.6 $\left.\mathrm{MHz}, \mathrm{CDCl}_{3}\right) \delta 30.2\left(\mathrm{CH}_{3}\right), 33.4\left(\underline{\mathrm{C}}\left(\mathrm{CH}_{3}\right)_{3}\right), 116.2\left(=\underline{\mathrm{CHC}}\left(\mathrm{CH}_{3}\right)_{3}\right), 124.3(\mathrm{Ar}), 126.3(\mathrm{Ar}), 130.5$ (C3), 131.7 (Ar), 146.9 (C5), 151.8 (C4), 161.0(Ar), 165.1 (Ar), 168.7 (C2). UV ( $\left.\mathrm{CH}_{3} \mathrm{OH}\right) \lambda_{\max }$ $202 \mathrm{~nm}\left(\varepsilon 13885 \mathrm{M}^{-1} \mathrm{~cm}^{-1}\right), 281$ (16941). IR (KBr) v $v_{\max } 2961,1764,1490,1261,1234,1082,977$, $830,770 \mathrm{~cm}^{-1}$. HRMS (ESI) $\mathrm{m} / \mathrm{z}$ calcd for $\mathrm{C}_{15} \mathrm{H}_{15} \mathrm{FO}_{2} \mathrm{Na}(\mathrm{M}+\mathrm{Na})^{+} 269.0954$, found 269.0975 . 


\section{Acknowledgements}

Financial support from the Australian Research Council is gratefully acknowledged.

\section{References and Notes}

1. Alanis, A. J. Arch. Med. Res. 2005, 36, 697.

2. Livermore, D. M. Clin. Infect. Dis. 2003, 36, S11.

3. Fuqua, W. C.; Winans, S. C.; Greenberg, E. P. J. Bacteriol. 1994, 176, 269.

4. Rice, S. A.; McDougald, D.; Kumar, N.; Kjelleberg, S. Curr. Opin. Investig. D. 2005, 6, 178.

5. Parsek, M. R.; Val, D. L.; Hanzelka, B. L.; Cronan, J. E., Jr.; Greenberg, E. P. Proc. Natl. Acad. Sci. USA 1999, 96, 4360.

6. Givskov, M.; de Nys, R.; Manefield, M.; Gram, L.; Maximilien, R.; Eberl, L.; Molin, S.; Steinberg, P. D.; Kjelleberg, S. J. Bacteriol. 1996, 178, 6618.

7. Manefield, M.; de Nys, R.; Kumar, N.; Read, R.; Givskov, M.; Steinberg, P.; Kjelleberg, S. Microbiology 1999, 145, 283.

8. Hentzer, M.; Riedel, K.; Rasmussen, T. B.; Heydorn, A.; Andersen, J. B.; Parsek, M. R.; Rice, S. A.; Eberl, L.; Molin, S.; Hoiby, N.; Kjelleberg, S.; Givskov, M. Microbiology 2002, 148, 87.

9. Hentzer, M.; Wu, H.; Andersen, J. N.; Riedel, K.; Rasmussen, T. B.; Bagge, N.; Kumar, N.; Schembri, M. A.; Song, Z.; Kristoffersen, P.; Manefield, M.; Costerton, J. W.; Molin, S.; Eberl, L.; Steinberg, P.; Kjellberg, S.; Hoiby, N.; Givskov, M. EMBO 2003, 22, 3803.

10. Wu, H.; Song, Z.; Hentzer, M.; Andersen, J. B.; Molin, S.; Givskov, M.; Hoiby, N. J. Antimicrob. Chemoth. 2004, 53, 1054.

11. de Nys, R.; Givskov, M.; Kumar, N.; Kjelleberg, S.; Steinberg, P. D. In Antifouling compounds; Fusetani, N., Clare, A. S., Ed.; Springer-Verlag: Berlin, Heidelberg, 2006; Vol. 42, pp 55-85.

12. Alvi, K. A., Casey, A., Nair, B. G. J. Antibiot. 1997, 51, 515.

13. Bouzard, D.; Di Cesare, P.; Essiz, M.; Jacquet, J. P.; Remuzon, P.; Weber, A.; Oki, T.; Masuyoshi, M. J. Med. Chem. 1989, 32, 537.

14. Klopman, G.; Fercu, D.; Renau, T. E.; Jacobs, M. R. Antimicrob. Agents Ch. 1996, 40, 2637.

15. Miyaura, N.; Suzuki, A. Chemical Reviews 1995, 95, 2457.

16. Song, Y. S.; Lee, Y.-J.; Kim, B. T.; Heo, J.-N. Tetrahedron Lett. 2006, 47, 7427.

17. Boukouvalas, J.; Lachance, N.; Ouellet, M.; Trudeau, M. Tetrahedron Lett. 1998, 39, 7665.

18. Kumar, N.; Read, R., PCT Int. Appl. AU02/00781, 2002.

19. Sorg, A.; Siegel, K.; Brückner, R. Synlett 2004, 0321.

20. Willis, C.; Bodio, E.; Bourdreux, Y.; Billaud, C.; Gall, T. L.; Mioskowski, C. Tetrahedron Lett. 2007, 48, 6421.

21. Jakubec, P.; Berkeš, D; Kolarovič, A; Považanec, F. Synthesis 2006, 23, 4032.

22. Kawashima, M.; Sato T.; Fujisawa, T. B. Chem. Soc. Jpn. 1988, 61, 3255. 